\title{
Papers from the Ribeirão Preto and São Luís birth cohort studies. Presentation
}

The present issue reports data for three cohort studies carried out in Brazil during the last three decades. Two were conducted in Ribeirão Preto, São Paulo, a developed city located in the Brazilian Southeast, and one in São Luís, Maranhão, a less developed city located in the Brazilian Northeast. The first cohort study, entitled "Epidemiological-social study of perinatal health in Ribeirão Preto" was conducted on all liveborn infants delivered at hospitals in Ribeirão Preto during one year from June 1978 to May 1979. The objective was to construct an extensive field of investigation of health aspects related to human reproduction for the analysis of the health-sickness process and of medical care and their consequences for infant mortality and for mortality during other stages of life. The mothers who participated in the study were interviewed after delivery and data regarding the newborn infants were obtained from the medical records. The information regarding the project is contained in 9067 questionnaires corresponding to approximately $98 \%$ of the universe of mother-liveborn infant pairs delivered at all maternities in the city, 6827 of which correspond to singleton deliveries by mothers from the municipality. This was the first study of its kind in Brazil, in which health aspects were related to the perinatal period.

This approach was repeated by locating the children at school age (1987/1989), by creating the conditions for a similar project in 1994 in order to carry out comparative studies, by reevaluating the boys of the cohort in $1996 / 97$ at the time of military service, and by studying mortality up to 20 years of age.

In 1994, the second cohort study was carried out in Ribeirão Preto, when information was obtained about a sample of one third of the births that had occurred at all maternities of the municipality in that year (3663 births, 2846 of which corresponding to singleton deliveries by mothers from the municipality) during their different phases of execution. Their success motivated the development of the third cohort described in this issue, in São Luís in 1997/98, resulting in a series of comparative analyses between the two cities. In 2004, these studies were complemented with a thematic project in which samples of individuals from the two cohorts of Ribeirão Preto (1978/79 and 1994) and the cohort of São Luís (1997/98) were evaluated together. The first Ribeirão Preto cohort 
was reevaluated at 23/25 years of age and the second Ribeirão Preto cohort and the São Luís cohort were reevaluated simultaneously at 7/11 years of age. The objective of the follow-up of these cohorts was to evaluate the contribution of early events in the life cycle to somatic growth and to the risk of development of non-communicable chronic diseases among young adults. In this respect, special attention was devoted to the impact of birth size on the physical, cognitive and psychological development of the children at school age.

Twelve articles comparing the 1978/79 and 1994 Ribeirão Preto cohorts and geographical comparisons between the 1994 Ribeirão Preto cohort and the 1997/98 São Luís cohort are presented in this issue of the Brazilian Journal of Medical and Biological Research, in addition to some preliminary data for the 1978/79 cohort up to adult age. This publication provides the opportunity to synthesize the results of an investigation that has been ongoing for 30 years.

Marco Antonio Barbieri Heloisa Bettiol Antônio Augusto Moura da Silva Marcelo Zubaran Goldani 